\section{Confiabilidade teste-reteste do item único de saúde bucal percebida em uma população de adultos no Rio de Janeiro, Brasil}

\author{
Test-retest reliability of self-perceived oral health \\ in an adult population in Rio de Janeiro, Brazil
}

\author{
${ }_{1}^{1}$ Agência Nacional de Saúde \\ Suplementar, Rio de Janeiro, \\ Brasil. \\ 2 Instituto de Medicina \\ Social, Universidade do \\ Estado do Rio de Janeiro, \\ Rio de Janeiro, Brasil. \\ 3 Escola Nacional de Saúde \\ Pública Sergio Arouca, \\ Fundação Oswaldo Cruz, \\ Rio de Janeiro, Brasil. \\ Correspondência \\ P. Nadanovsky \\ Instituto de Medicina Social, \\ Universidade do Estado \\ do Rio de Janeiro. \\ Rua São Francisco Xavier \\ 524, $7^{\circ}$ andar, Blocos D e E, \\ Rio de Janeiro, $R J$ \\ 20559-900, Brasil. \\ nadanovsky@ims.uerj.br
}

\begin{abstract}
This study evaluated the test-retest reliability of a single item, namely self-perceived oral health, included in a questionnaire applied in a longitudinal study (the "Pró-Saúde" or Pro-Health Study, 2001). The questionnaire was applied twice in a sample of 101 employees at a State university in Rio de Janeiro, Brazil. Self-perceived oral health was based on a single item, with five choices, ranging from "very good" to "very bad". Agreement was estimated using weighted kappa statistics and stratified according to gender, age, income, and schooling. Overall kappa coefficient was 0.80. Higher values were found in women ( $k=0.84)$, young adults ( $k=0.85)$, subjects with medium-level schooling ( $k=0.86)$, and those earning more than six times the minimum wage $(k=0.91)$. Lower kappa estimates were obtained for individuals over 40 years of age ( $k=0.67$ for $40-49$ years and $k=$ 0.69 for $\geq 50$ ). The reliability of the single item "self-perceived oral health" varied from "considerable" to "almost perfect" in all population strata, suggesting that this item can be used in future analyses in the scope of the "Pró-Saúde" Study.
\end{abstract}

Oral Health; Questionnaires; Reproducibility of Results

\author{
Gislaine Afonso-Souza ${ }^{1}$ \\ Paulo Nadanousky ${ }^{2}$ \\ Guilherme Loureiro Werneck 2 \\ Eduardo Faerstein 2 \\ Dora Chor 3 \\ Cláudia S. Lopes 2
}

\section{Introdução}

Pesquisas sobre saúde bucal percebida têm tido como objetivo desenvolver instrumentos de mensuração que sejam capazes de captar perspectivas sobre saúde bucal que vão além do que medidas clínicas tradicionais são capazes de captar 1,2,3,4,5, ou seja, instrumentos que incorporem a percepção do indivíduo sobre sua própria saúde bucal 5,6,7.

Um desses instrumentos é o "item único de saúde bucal percebida”. A grande riqueza dessa medida é ilustrada pela forte associação com questionários relevantes para a saúde bucal, incluindo questões relativas à estética, mastigação, conforto, bem-estar psicológico, relacionamento social, qualidade de vida geral e bucal 1,2,3,4,5,8,9 e saúde geral 4 .

A avaliação da qualidade das informações produzidas em estudos epidemiológicos é crucial para a garantia de que os resultados obtidos reflitam os elementos teóricos que se pretende captar. Em estudos que utilizam questionários autopreenchíveis, como o aplicado no Estudo Pró-Saúde 10, busca-se avaliar quão reprodutíveis são as informações, quando obtidas em diferentes momentos do tempo sob circunstâncias similares (confiabilidade teste-reteste).

O presente estudo tem como principal objetivo avaliar a confiabilidade teste-reteste do item único de saúde bucal percebida na população do Estudo Pró-Saúde como um todo e segundo sexo, 
faixa etária, nível de escolaridade e renda familiar per capita.

\section{Métodos}

Um questionário multidimensional autopreenchível foi aplicado em junho de 2001 para a Fase 2 do Estudo Pró-Saúde 10 (estudo aprovado pelo Comitê de Ética da Universidade do Estado do Rio de Janeiro 11). Uma amostra de conveniência de 101 funcionários foi utilizada para o estudo de confiabilidade teste-reteste. O questionário foi aplicado duas vezes, com intervalo de uma semana entre as duas aplicações.

O item único de saúde bucal percebida foi assim apresentado: "De um modo geral, como você considera o seu estado de saúde bucal (dentes e gengiva)?", com cinco opções de resposta, "muito bom", "bom", "regular", "ruim" e "muito ruim”. Para efeito de análise estatística, o item único de saúde bucal percebida foi considerado nas cinco categorias originais e em duas formas binárias: (i) as respostas "muito bom" e "bom" agregadas em uma categoria, e "regular", "ruim" e "muito ruim" em outra; (ii) "muito bom", "bom" e "regular" agregadas em uma categoria, e "ruim" e "muito ruim" em outra.

A avaliação da confiabilidade teste-reteste para toda a população e segundo sexo, faixa etária, níveis de escolaridade e renda familiar per capita, foi baseada em estimativas da estatística kappa (k), ponderadas com pesos quadráticos, já que o item único de saúde bucal percebida é um indicador multicategórico ordinal. Nas formas binárias da saúde bucal percebida, utilizamos estimativas da estatística kappa não ponderada (k) 12,13. Para todas as estatísticas foram estimados intervalos de 95\% de confiança (IC95\%), calculados através de procedimentos de bootstrap, com mil replicações 14. Para essas análises, utilizou-se o programa estatístico Stata 7.0 (Stata Corporation, College Station, Estados Unidos).

\section{Resultados}

A população participante apresentou ligeira predominância do sexo feminino (53\%) e idade variando entre 19 e 66 anos (média de 36 anos, desvio-padrão de 12,4). A maior parte dos funcionários apresentava o $2^{\circ}(47 \%)$ ou $3^{\circ}$ grau completo ou mais (39\%), sendo que $61 \%$ tinham renda per capita menor ou igual a 3 salários mínimos (1 salário mínimo em 2001 equivalia a $\mathrm{R} \$ 180,00)$.

Em relação a toda população, encontrou-se um coeficiente kappa ponderado quadrático de 0,75 a 0,80 (Tabela 1). A estabilidade desse indicador, nas diferentes formas de categorização, é substancial 15. Valores pontuais mais altos foram obtidos para mulheres $(k=0,84$; IC95\%: 0,71-0,94); adultos jovens na faixa etária entre 30 e 39 anos ( $k=0,85$; IC95\%: 0,55-0,97); participantes com nível médio de escolaridade ( $\mathrm{k}=$ 0,86; IC95\%: 0,74-0,95) e os com renda maior que

Confiabilidade teste-reteste do item único de saúde bucal percebida. Estudo Pró-Saúde, Rio de Janeiro, Brasil, 2001.

\begin{tabular}{|c|c|c|c|c|c|c|c|c|c|}
\hline \multirow[t]{2}{*}{ Saúde bucal percebida } & \multicolumn{3}{|c|}{++} & \multicolumn{3}{|c|}{+-} & \multirow[t]{2}{*}{ Concordância (\%) } & \multirow[t]{2}{*}{ k } & \multirow[t]{2}{*}{ IC95\% } \\
\hline & & -+ & & & -- & & & & \\
\hline 5 categorias * & & A & $B$ & C & $\mathrm{D}$ & $E$ & 78,49 & 0,80 & $0,69-0,89$ \\
\hline$A=$ Muito bom & A & 12 & 4 & 0 & 0 & 0 & & & \\
\hline $\mathrm{B}=\mathrm{Bom}$ & B & 5 & 38 & 3 & 1 & 0 & & & \\
\hline$C=$ Regular & C & 0 & 6 & 19 & 1 & 0 & & & \\
\hline$D=$ Ruim & $\mathrm{D}$ & 0 & 0 & 0 & 4 & 0 & & & \\
\hline $\mathrm{E}=$ Muito ruim & $E$ & 0 & 0 & 0 & 0 & 0 & & & \\
\hline 2 categorias ** & & & & & & & 89,25 & 0,75 & $0,60-0,90$ \\
\hline Muito bom/Bom vs. & & 59 & & & 4 & & & & \\
\hline Regular/Ruim/Muito ruim & & 6 & & & 24 & & & & \\
\hline 2 categorias ** & & & & & & & 97,85 & 0,79 & $0,51-1,00$ \\
\hline Muito bom/Bom/Regular vs. & & 87 & & & 2 & & & & \\
\hline Ruim/Muito ruim & & 0 & & & 4 & & & & \\
\hline
\end{tabular}

* Coeficiente kappa ponderado quadrático;

** Coeficiente kappa não ponderado. 
seis salários mínimos ( $\mathrm{k}=$ 0,91; IC95\%: 0,68-1,00) (Tabela 2).

\section{Discussão}

De uma forma geral, os resultados encontrados neste estudo indicam que a informação obtida por meio do item único de saúde bucal percebida tem estabilidade variando entre substancial e quase perfeita $(0,75$ e 0,80$)$ ou, no mínimo, moderada, ao se tomar o valor do limite inferior do intervalo de confiança $(0,51)$ da segunda forma de categorização das opções de resposta. $\mathrm{O}$ mesmo ocorre para os diferentes estratos populacionais, sendo que, em termos de valores dos limites inferiores, a estabilidade é pobre $(0,11 \mathrm{e}$ 0,19 ) para os indivíduos com $1^{\circ}$ grau completo e acima de 50 anos ( $p>0,05)$; ou estabilidade regular $(0,36)$ para indivíduos com idade entre 40 e 49 anos. Esse resultado pode ser explicado pela baixa prevalência de indivíduos nessas categorias, particularmente os de baixa escolaridade e mais velhos. Em termos pontuais, os melhores resultados foram obtidos para as mulheres, adultos jovens e os que possuíam nível de educação e renda mais altos. As pequenas diferenças de concordância encontradas nas diferentes faixas etárias e em homens e mulheres não devem ser atribuídas, primordialmente, a diferenciais de compreensão ou interpretação do item único de saúde bucal percebida, já que se trata de uma população em que a grande maioria (86\%) dos indivíduos tem bom nível instrucional. Observou-se também, que não houve informações ausentes nas duas aferições, sugerindo clareza de linguagem e boa compreensão do item.

Especula-se que, de uma forma geral, as mulheres apresentem melhor percepção de suas condições bucais 16 , tenham melhor comportamento relacionado à saúde e buscam mais tratamento 17. Por isso, talvez sejam mais conscientizadas em relação à saúde bucal, o que as favorece em termos de consistência nas respostas relativas a essa questão, em comparação aos homens. Estudos referentes à confiabilidade teste-reteste de questões como eventos de vida estressantes 18 , história de diagnóstico e tratamento de hipertensão 10 e aspectos da rede social 11, realizados no Estudo Pró-Saúde, além de apresentarem estabilidade satisfatória ( $\mathrm{k}$ entre 0,62 e 1,00; $\mathrm{k}=0,75$, IC95\%: 0,73-0,77; $\mathrm{k}$ acima de 0,70 para a maioria das variáveis, respectivamente), têm mostrado que as mulheres são mais consistentes em su-

Tabela 2

Confiabilidade teste-reteste do item único de saúde bucal percebida, segundo sexo, idade, escolaridade e renda. Estudo Pró-Saúde, Rio de Janeiro, Brasil, 2001.

\begin{tabular}{|c|c|c|c|}
\hline & \multirow[t]{2}{*}{$\mathbf{n}$} & \multicolumn{2}{|c|}{ Saúde bucal percebida } \\
\hline & & k * & IC95\% \\
\hline \multicolumn{4}{|l|}{ Sexo } \\
\hline Feminino & 49 & 0,84 & $0,71-0,94$ \\
\hline Masculino & 44 & 0,74 & $0,52-0,89$ \\
\hline \multicolumn{4}{|l|}{ Grupo etário (anos) } \\
\hline $19-29$ & 40 & 0,84 & $0,69-0,96$ \\
\hline $30-39$ & 18 & 0,85 & $0,55-0,97$ \\
\hline $40-49$ & 20 & 0,67 & $0,36-0,91$ \\
\hline$>50$ & 15 & 0,69 & $0,19-1,00$ \\
\hline \multicolumn{4}{|l|}{ Escolaridade } \\
\hline $1^{\circ} \mathrm{grau}$ incompleto & 3 & - & - \\
\hline $1^{\circ}$ grau completo & 7 & 0,79 & $0,11-1,00$ \\
\hline $2^{\circ}$ grau completo & 44 & 0,86 & $0,74-0,95$ \\
\hline $3^{\circ}$ grau ou mais & 35 & 0,81 & $0,63-0,92$ \\
\hline \multicolumn{4}{|c|}{ Renda per capita (salários mínimos) } \\
\hline$<3$ & 57 & 0,75 & $0,56-0,89$ \\
\hline $3-6$ & 24 & 0,85 & $0,64-0,97$ \\
\hline$>6$ & 11 & 0,91 & $0,68-1,00$ \\
\hline Total & 93 & & \\
\hline
\end{tabular}

* Coeficiente kappa não ponderado. 
as respostas. Entretanto, o estudo de Vieira 9 em uma população de puérperas, cujas informações foram coletadas por meio de entrevista, apresentou um coeficiente kappa para o item único de saúde bucal percebida moderado $(\mathrm{k}=0,58$; IC95\%: 0,38-0,77). Talvez pelo fato dessa população ser predominantemente de baixa escolaridade e renda possa explicar de alguma forma o coeficiente de concordância somente moderado. Em nosso estudo, a grande maioria (94\%) das mulheres possuía $2^{\circ}$ e $3^{\circ}$ graus completos ou mais de escolaridade, o que parece reforçar mais ainda a estabilidade, pois o nível de escolaridade 19 pode afetar a compreensão da pergunta e, conseqüentemente, a resposta. No caso de indivíduos mais velhos, pode-se pensar que, devido ao declínio do estado bucal ao longo da vida, eles estariam mais freqüentemente sujeitos a problemas bucais (doença periodontal, exodontias, entre outros) do que indivíduos mais jovens. Nesse caso, a prevalência de sintomas associados a esses problemas (por exemplo, o aumento de dor dental) poderia ter provocado certa discordância entre as duas medidas. Níveis de escolaridade e renda são fatores importantes, dado que altos níveis são facilitadores de acesso à informação e aos serviços de saúde em geral, além de proporcionar maior habilidade cognitiva. Por outro lado, não se pode excluir a hipótese de que as pequenas variações das estimativas de concordância entre estratos populacionais decorram meramente de variações na prevalência da saúde bucal percebida nos subgrupos populacionais. Nesse caso, subgrupos com maior ou menor prevalência de respostas positivas tenderiam a ter valores de kappa aumentados 20.

Análises futuras utilizando-se o item único de saúde bucal percebida serão feitas com a forma de agregação em que a categoria "regular" foi agregada às opções de respostas "muito bom" e "bom", pois os achados (Tabela 1) parecem indicar que, do ponto de vista qualitativo, para os indivíduos desta população a categoria "regular" está mais próxima da categoria "bom" do que da categoria "ruim".

O intervalo de tempo de uma semana entre as duas medidas foi pertinente em nosso estudo, pois o item único de saúde bucal percebida foi aferido utilizando-se uma pergunta genérica sobre saúde bucal, que não fez referência a um intervalo de tempo específico.

O estudo indica que o item único de saúde bucal percebida pode ser usado em análises futuras do Estudo Pró-Saúde, dado que os coeficientes de confiabilidade foram satisfatórios para todos os estratos populacionais estudados e por sua forte associação, em outros estudos 1,2,3,4,5,8,9, com questionários relevantes para a saúde bucal. Apesar de a confiabilidade do item único de saúde bucal percebida ter apresentado pequenas variações de acordo com o sexo, idade, escolaridade e renda, esta variação parece não ser substancial a ponto de inviabilizar seu uso neste contexto. Porém, a generalização dos nossos achados deve ser limitada, por se tratar de uma população socioeconomicamente mais favorecida do que a população do Rio de Janeiro e do Brasil em geral. 


\section{Resumo}

O estudo avaliou a confiabilidade teste-reteste do item único de saúde bucal percebida, que fez parte de um questionário de um estudo longitudinal (Estudo PróSaúde, 2001). Esse questionário foi aplicado duas vezes, em uma amostra de 101 funcionários de uma universidade do Rio de Janeiro, Brasil. A avaliação da saúde bucal percebida foi feita utilizando-se um item único com cinco opções de resposta: de "muito bom" a "muito ruim". A concordância foi estimada pela estatística kappa (k) ponderada quadrática e estratificada segundo sexo, idade, renda e escolaridade. O coeficiente kappa para toda população foi 0,80. Valores pontuais mais altos foram obtidos para mulheres $(k=0,84)$, adultos jovens $(k=0,85)$, participantes com nível médio de escolaridade $(k=0,86)$ e os de renda maior que seis salários mínimos $(k=0,91)$. Estimativas kappa mais baixas foram encontradas em indivíduos acima de 40 anos ( $k=0,67,40-49$ anos; $e k=0,69, \geq 50$ ). $A$ confiabilidade do item único de saúde bucal percebida variou de substancial a quase perfeita, para todos os estratos da população, sugerindo que este item pode ser usado em análises futuras no âmbito do Estudo Pró-Saúde.

Saúde Bucal; Questionários; Reprodutibilidade dos Testes

\section{Colaboradores}

G. Afonso-Souza participou da análise dos dados e redação do artigo. P. Nadanovsky e G. L. Werneck orientaram a redação e revisaram o artigo. E. Faerstein, D. Chor e C. S. Lopes contribuíram para a coleta dos dados e revisão da redação do artigo.

\section{Agradecimentos}

A Dra. Gislaine Afonso-Souza recebeu apoio da Fundação de Amparo à Pesquisa do Estado do Rio de Janeiro (FAPERJ), processo no. E-26/150.889/2001. Este artigo faz parte da Tese de Doutorado O Item Único de Saúde Bucal Percebida - Confiabilidade Teste-reteste e a Associação com a Visita Odontológica para Revisão de Rotina - em uma População de Adultos no Rio de Janeiro: Evidência do Estudo Pró-Saúde, defendida no Instituto de Medicina Social, Universidade do Estado do Rio de Janeiro em 2005.

\section{Referências}

1. Atchison KA, Dolan TA. Development of the Geriatric Oral Health Assessment Index. J Dent Educ 1990; 54:680-7.

2. Atchison KA, Matthias RE, Dolan TA, Lubben JE, De Jong F, Mayers-Oakes AS. Comparison of oral health rating by dentists and dentale elders. J Public Health Dent 1993; 53:223-30.

3. Cushing AM, Sheiham A, Maizels J. Developing socio-dental indicators - the social impact of dental disease. Community Dent Health 1986; 3:3-17.

4. Matthias RE, Atchison KA, Lubben JE, De Jong F, Schweitzer SO. Factors affecting self-ratings of oral health. J Public Health Dent 1995; 55:197-204.

5. Slade GD, Spencer AJ. Development and evaluation of the Oral Health Impact Profile. Community Dent Health 1994; 11:3-11.

6. Locker D. Measuring oral health: a conceptual framework. Community Dent Health 1988; 5:3-18.
7. Rosenberg D, Kaplan S, Senie R, Badner V. Relationships among dental functional status, clinical dental measures, and generic health measures. J Dent Educ 1988; 52:653-7.

8. Locker D, Miller Y. Evaluation of subjective oral health status indicators. J Public Health Dent 1994; 54:167-76.

9. Vieira BHOM. Prevalência e impacto da dor de dente em uma população de mulheres grávidas no Rio de Janeiro, Brasil [Tese de Doutorado]. Rio de Janeiro: Instituto de Medicina Social, Universidade do Estado do Rio de Janeiro; 2003.

10. Faerstein E, Chor D, Lopes CS. Reliability of the information about the history of diagnosis and treatment of hypertension. Differences in regard to sex, age, and educational level. The Pro-Saude Study. Arq Bras Cardiol 2001; 76:301-4. 
11. Griep RH, Chor D, Faerstein E, Lopes C. Confiabilidade teste-reteste de aspectos da rede social no Estudo Pró-Saúde. Rev Saúde Pública 2003; 37:37985.

12. Cohen JA. Coefficient of agreement for nominal scales. Educ Psychol Meas 1960; 20:37-46.

13. Cohen JA. Weighted kappa: nominal scale agreement with provision for scaled disagreement or partial credit. Psychol Bull 1968; 70:213-20.

14. Efron B, Tibshirani R. An introduction to the Bootstrap. London: Chapman and Hall; 1993.

15. Landis JR, Koch GG. The measurement of observer agreement for categorical data. Biometrics 1977; 33:159-74.

16. Ostberg AL, Lindblad U, Halling A. Self-perceived oral health in adolescents associated with family characteristics and parental employment status. Community Dent Health 2003; 20:159-64.
17. Reisine S, Bailit HL. Clinical oral health status and adult perceptions of oral health. Soc Sci Med 1980; 14A:597-605.

18. Lopes CS, Faerstein E. Confiabilidade do relato de eventos de vida estressantes em um questionário autopreenchido: Estudo Pró-Saúde. Rev Bras Psiquiatr 2001; 23:126-33.

19. Chor D, Faerstein E, Alves MG, Lopes CS. How reproducible is self-reported information on exposure to smoking, drinking, and dietary patterns? Evidence among Brazilian adults in the Pró-Saúde Study. São Paulo Med J 2003; 121:63-6.

20. Feinstein AR, Cicchetti DV. High agreement but low kappa: I. The problems of two paradoxes. J Clin Epidemiol 1990; 43:543-9.

Recebido em 01/Fev/2006

Versão final reapresentada em 14/Nov/2006 Aprovado em 31/Jan/2007 